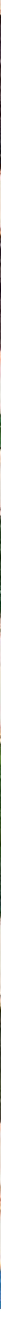

\title{
The adventure of quasicrystals: a sucessful multidisciplinary effort
}

• D. Gratias - LEM CNRS-ONERA, BP 72, 29 avenue de la Division Leclerc, 92322 Châtillon Cedex - DOI: 10.1051/epn/2012502

The 2011 Nobel Prize for chemistry was awarded to Daniel Shechtman from the Technion Haifa (Israel) for his discovery of quasicrystals in 1982. At the time, he was visiting the National Bureau of Standards (now NIST, Gaithersburg, Maryland, USA). While performing an electron microscopy study of rapidly solidified aluminium alloys, he observed precipitates with beautifully resolved sharp diffraction spots with 5-fold symmetry as shown on fig 1 (a). His surprise was such that he initially refused to publish this unbelievable result.

$\triangle$ Tile decoration, Alhambra Palace, Grenade, Spain. It is not a quasicrystal

structure but

makes a pleasant illustration related to it. @ iStockPhoto

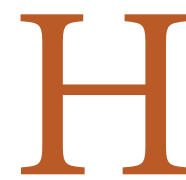
first wanted to find an explanation for this paradoxical result of an object diffracting like a perfect crystal but with overall 5-fold Dan said: "there is no such animal". It was only in late spring1984 that Ilan Blech, one of his colleague at Technion, devised a very clever model of random stacking of parallel regular icosahedra linked by the edges and that showed strong diffraction peaks at the wavevector positions observed by Shechtman.

At about the same time, Shechtman consulted John Cahn, Senior scientist at NIST (NBS), who suggested him to focus on the experimental fact of the paradox of Bragg diffraction showing a non-crystallographic symmetry in a solid. Shechtman invited Cahn to write such common paper that eventually became the PRL paper with the 
help of Gratias who revealed the work of $\mathrm{H}$. Bohr and A. Besicovic on almost-periodicity, thus confirming the possible existence of Bragg diffraction for non-periodic objects. This led to the two papers [1] that announced the discovery of quasicrystals.

\section{Prolegomena of quasicrystals}

As very often in science, the concepts that were at the basis of quasicrystals were already known for a long time! In 1972, Yves Meyer [2] published a book on Harmonic Analysis and Number Theory in which he invented the so-called harmonious ensembles that are non-periodic sets of points that diffract on enumerable sets of Bragg peaks. These harmonious ensembles are now recognized as the first quasicrystals. In 1977 Pieter Marten de Wolff, followed by Aloysio Janner and Ted Janssen [3], invented the superspace description for describing incommensurate phases. A few years before, in 1974, Roger Penrose built a non-periodic tiling made of two kinds of tiles with an overall 5-fold symmetry [4]. In 1981, Alan L. Mackay choose the Penrose tilings as prototypes of possible atomic distributions with 5 -fold symmetries in a magnificent pioneering paper [5] De Nive Quiquangula: on the pentagonal snowflake where he concluded: "it gives an example of a pattern of the type which might well be encountered but which might go unrecognized if unexpected". The link between the Penrose tiling and the superspace description started with the 1981 paper Algebraic theory of Penrose's non-periodic tilings of the plane by Nicolaas Govert de Bruijn [6] who discovered the hidden properties of this tiling in considering internal variables that would later be shown as the basis of the cut method. Soon after, in 1984, Peter Kramer and Roberto Neri [7] found new periodic and non-periodic space fillings by projection from higher dimension spaces. Finally, a few weeks after Shechtman's publication, Dov Levine and Paul Steinhardt [8] proposed the first explicit ideal structural model that they designated as quasicrystals, an abbreviation for quasiperiodic crystals.

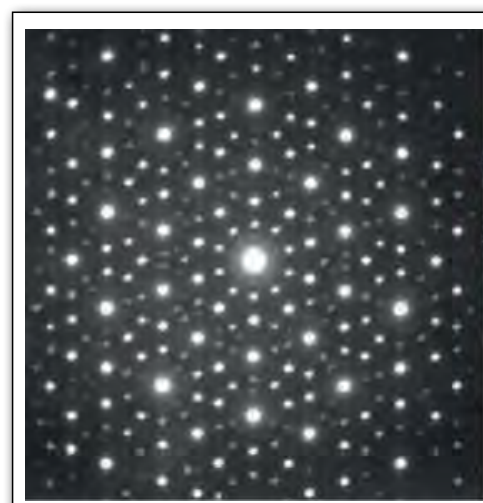

(a)

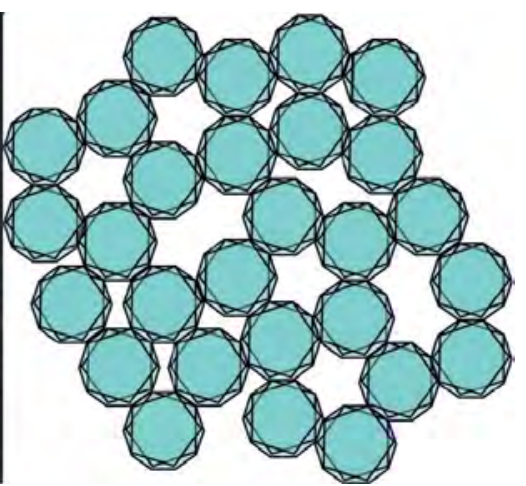

(b)
$\Delta$ FIG. 1. (a) Electron Diffraction pattern of dendritic precipitates in rapidly quenched AI6Mn alloy showing a 5 -fold symmetry forbidden by crystallography. (b) Ilan Blech's first multipolyhedral model of randomly distributed Al12Mn parallel icosahedra connected by the edges.

\section{The basics of quasiperiodicity}

The common feature between these theoretical works and Shechtman's experiments is the fact that symmetries are hidden in a higher dimension space. This is best understood in the reciprocal space: the diffraction patterns like Fig. 1(a) have the remarkable property that all the diffraction spots $q$ can be indexed using 6 integers $n_{i}, q=$ $\sum n_{i} e_{i}$, with unit vectors $e_{i}$ defined by the six 5-fold axes of the icosahedron. So the diffraction spots can all be viewed as the projections $q$ in the physical 3-dim space, of 6-dim vectors $Q$ of a regular periodic (reciprocal) lattice in a 6-dim Euclidian space. In direct space, this corresponds to an irrationally oriented cut of a periodic object in a 6-dim space. This is the definition of quasiperiodicity: a quasiperiodic pattern in a $D$-dim space is the result of a $D$-dim irrationally oriented cut of an $\mathrm{N}$-dim periodic pattern with $N>D$ as illustrated on Fig. 3(a) and (b).

\section{Quasicrystals immediately accepted}

In January 1985, Louis Michel and Marjorie Senechal organised a workshop of mathematical crystallography at the Institut des Hautes Etudes Scientifiques (IHES- Bures/Yvette)

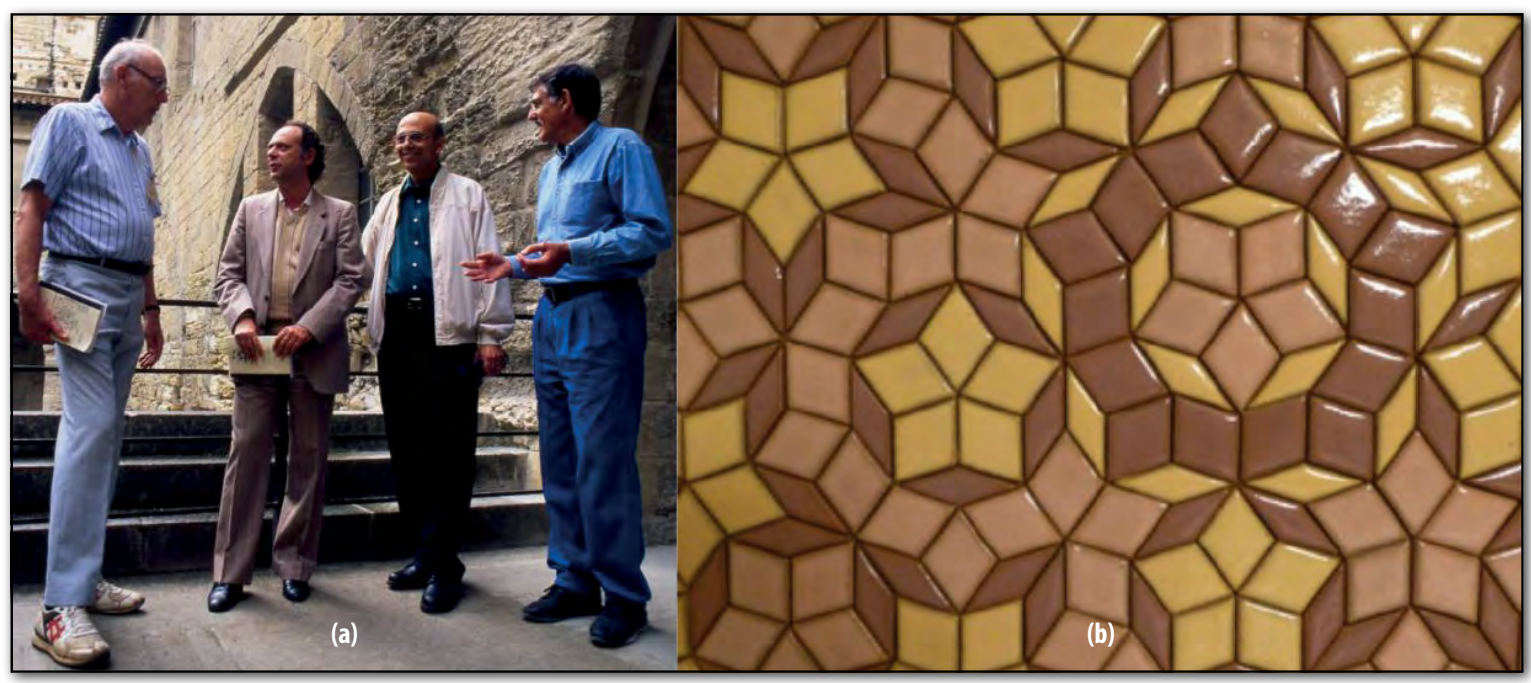

4 FIG. 2. (a) from right to left D. Shechtman, I. Blech, D. Gratias and J.W. Cahn (CNRSPhotothèquePierre Grumberg). (b) Example of a typical Penrose tiling showing local 5-fold symmetry. 


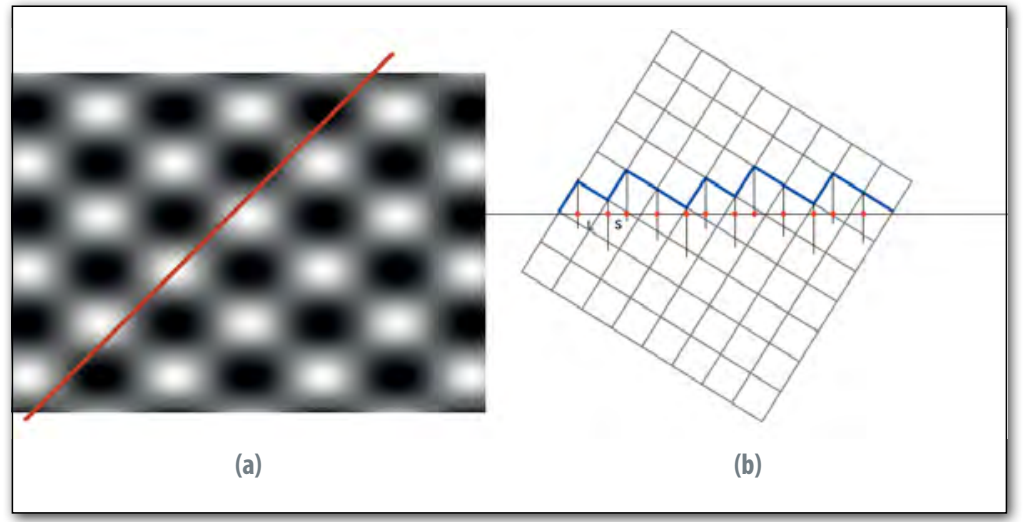

$\Delta$ FIG. 3. (a) The 1-dim quasiperiodic function $f(x)=\cos x+\cos \sqrt{2} x$ is the $(x=y)$ cut of the 2-dim periodic function $F(x, y)=\cos x+\cos \sqrt{2} y$. (b) Cutting a square lattice by a straight line of irrationnal slope $1 / \tau$ (where $\tau=(1+\sqrt{5}) / 2$ is the golden mean) and projecting the upperleft vertices of the intersected squares generates the (quasiperiodic) Fibonacci sequence of long (L) and short (S) segments.

near Paris and invited Shechtman, Cahn and Gratias to present their results. Michel Duneau, André Katz and Aloysio Janner were in the audience and immediately after Gratias's talk, Michel Duneau and André Katz [9] explained on the blackboard the technique of the cut method (see Fig. 3) whereas Aloysoi Janner made the connection with the superspace description: in that extraordinary afternoon session, the very bases of this new quasicrystallography were posed. The cut method has been independently confirmed by the Russian group Pavel Kalugin, Alexei Kitaev and Leonid Levitov [9] and by the American Veit Elser [9].

Quasicrystals received a unanimous enthousiastic interest from the mathematics and solid-state physics communities. The research developed very quickly in Europe: in France, with a joined research programme (PICS) with the NBS (Gaithersburg, Maryland, USA), the first International Workshop on Quasicrystals in Les Houches (March 1986), the creation of the Groupe de Recherche CNRS Quasicristaux untill the 90's, and then,

v FIG. 4. Typical example of quasiperiodic i-AICuFe structure made of an ordered imbrication at long distance of atomic clusters (shown here are the so-called Bergman clusters made of a central atom, an icosahedron and an external icosidodecahedron). A cut of the structure perpendicular to a 5 -fold direction shows that the clusters are connected by edges of the external icosidodecahedra like in the Blech random tiling model. As on the left, the clusters stack along the 5 -fold axes according to Fibbonacci sequences. The chemical species distribute on the various orbits of these clusters.

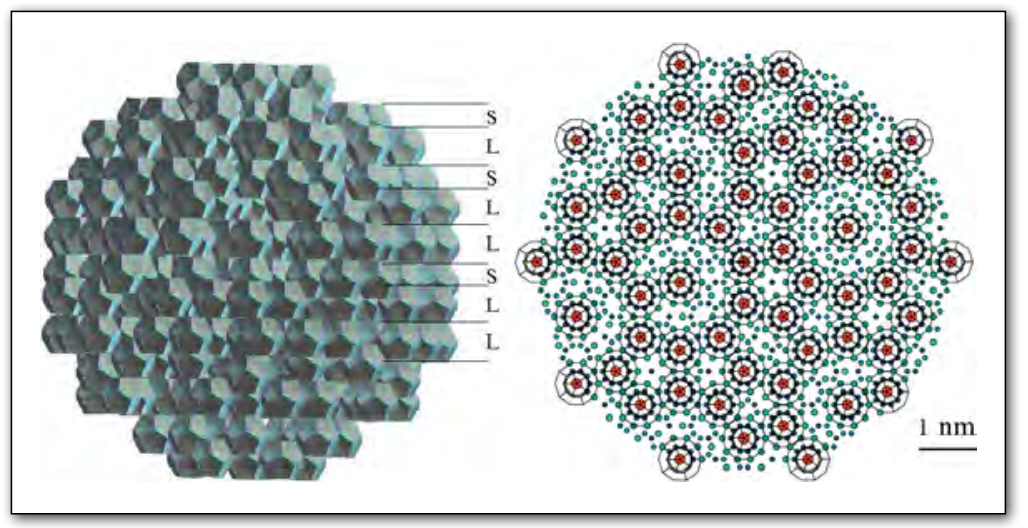

starting 1997, in Germany with the DFG Schwerpunkt Quasikristalle. Together, more than a hundred European laboratories have been involved in those programmes with quite comfortable financial support. Parts of these efforts are still very active today in a European Network of Excellence on Complex Metallic Alloys (CMA) that gathers 21 European Countries.

Although no scientific revolution has ever been easier than quasicrystals in Europe, the situation has been slightly different in the US. In fact, in a letter to Nature [10] in October 1985, the famous double Nobel Prize chemist and crystallographer Linus Pauling rebels against these quasicrystals that he thinks were simple multi-twinned ordinary crystals: "crystallographers can now cease to worry that the validity of one of the accepted bases of their science has been questioned". Strong refutations have immediately been published [10] in the January 1986 issue of Nature "Pauling's model not universally accepted" signed by several groups, but most of the US crystallographers stayed outside the field during many years. This has been the opportunity for European and Asiatic scientists to take some advance in sample preparation techniques and crystallography, whereas the US physicists developed mostly the entropy aspects with the random tilings models.

\section{Atomic structures of quasicrystals}

New quasicrystalline structures are rapidly discovered since 1985 : the decagonal phase is identified by L. Bendersky [11] at NIST, and then, a series of icosahedral metastable phases are found mostly in ternary aluminium-based alloys. In July 1986, P. Sainfort and B. Dubost, from the research center Cegedur-Péchiney near Grenoble, discover the first millimeter size stable quasicrystal [11] obtained by slow solidification in an Al-Li-Cu(Mg) alloy. Soon after, A.P. Tsai [11] finds an impressively large number of new stables icosahedral and decagonal phases.

This started the process of making quantitative X-ray and neutron single-grain diffraction studies. In November 1990 M. Cornier-Quiquandon et al. made a first structure determination of the $\mathrm{Al}_{62} \mathrm{Cu}_{25.5} \mathrm{Fe}_{12.5}$ single-grain icosahedral phase by neutron diffraction at LLB (CEA-Saclay, France). They used the cut and devised atomic surfaces inspired by previous work from P. Guyot et M. Audier, who proposed structural units (Mackay clusters) for quasicrystals from a study of the unit cells of the crystalline phases $\alpha-(\mathrm{AlFeSi})$ and $\alpha-(\mathrm{AlMnSi})$ (see Fig. 4). Independently, in 1992, M. Boudard et al. obtained very similar results on the alloy $\mathrm{Al}_{70.3} \mathrm{Pd}_{21.4} \mathrm{Mn}_{8.3}$ using X-ray diffraction at ESRF (Grenoble). In both cases, the result was surprisingly simple: the atomic structure, like the one shown on Fig. 4, can be described by three atomic surfaces located a the high-symmetry special points of the 6-dim lattice. 
The cut method is now successfully used on many other quasicrystalline icosahedral and decagonal phases including the most recently discovered phases in the $(\mathrm{Zn}, \mathrm{Mg}, \mathrm{Ho}),(\mathrm{Zn}, \mathrm{Sc}, \mathrm{Mn})$ and $(\mathrm{Cd}, \mathrm{Yb})$ systems.

\section{Physical properties}

Quasicrystals have very early on been recognized as HumeRothery phases with a pseudo gap near the Fermi level.A.P. Tsai - discoverer of almost all recent quasicrystals - has always been guided by the phenomenological criterion of the number of electrons per atom $(e / a)$ and the ratio of the covalent atomic radii of the alloy components to find new stable quasicrystals. Concerning transport properties, quasicrystals have very unique behaviour: their electrical resistivities at low temperature are several orders of magnitude larger than those of their components, similar to those of doped semiconductors. Moreover, contrary to usual metals, the resistivity increases with the quality of the quasicrystal and varies with temperature as an inverse Matthiesen rule! This phenomenon could be connected to a strong localisation of the electrons in the atomic clusters (see Fig. 4) and the many umklapp processes that could prevent electrons to jump from one cluster to the other. Similarly, quasicrystals have low thermal conductivity at low temperature. They could be used as coating in liquefied-gas containers. Also lattice dynamics of quasicrystals have very specific properties due to the existence of many neighbouring atomic sites that are mutually exclusive, thus generating phasons (analogy to incommensurate phases) that are discontinuous atomic jumps at finite distances, as first observed by quasi-elastic neutron scattering. It is now suggested that these atomic hopping modes are strongly dissipative, but the origin of this dissipation is still poorly understood. The mechanical properties of quasicrystals are very similar to those of complex intermetallics with large unit cells (Laves and/or Frank-Kasper phases). They are highly brittle at low temperature and ductile only close to their melting points. The dislocations in quasicrystals are defined in the $\mathrm{N}$-dim space in the same manner as for ordinary crystals, but their motions are restricted to high temperature where they move essentially by climb.

\section{As a conclusion...}

Quasiperiodicity does not exhaust, by far, all possible ordered solutions: many other mathematical deterministic algorithms exist that give rise to perfectly ordered distributions of points that are neither periodic nor quasiperiodic. It is not yet known which of these distributions could appear in Nature but, because of quasicrystals, it is clear that solid-state physicists are much better prepared to face these new possibilities today than in the 1980s. What is less clear is the present capability of the Europeans research agencies to quickly react and federate a multidisciplinary research in front of a hypothetical new "quasicrystal" discovery. -

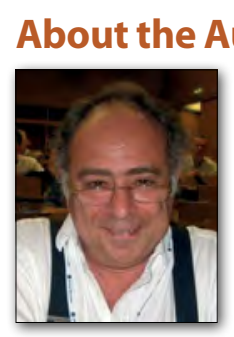

(n) Born in 1947, Denis Gratias received his diploma of the Ecole Nationale de Chimie de Paris in 1970 and made his Doctorate on the Crystallography of homogeneous interfaces in crystals in professor Michel Fayard's laboratory in Paris. He then worked with professor Louis Michel at the Institute of High Scientific Studies (IHES-Bure/ Yvette) and Professor Didier de Fontaine at the University of California Berkeley in statistical physics. Professor J. W. Cahn invited him to a longterm seminar at University California Santa-Barbara where he learned in summer 1984 about the astonishing results of D. Shechtman. He joined the group of Cahn at NIST and participated to the first paper announcing the discovery of quasicrystals. He then specialized in quasicrystal structure determination using $\mathrm{N}$-dim crystallography and he studies now the defects observed in quasicrystals like dislocations and the metadislocations in their approximants.

\section{References}

[1] D. Shechtman and I. Blech, Met Trans. 16A, 1005 (1985); D. Shechtman, I. Blech, D. Gratias and J. W. Cahn, PRL 53, 1951 (1984).

[2] Y. Meyer, Lectures notes in mathematics n 117, Springer (1970); ibid. Algebraic numbers and harmonic analysis, North-Holland (1972).

[3] P. M. deWolff, Acta Cryst. A 33, 493 (1977); A. Janner and T. Janssen, Phys. Rev. B 15, 643 (1977). See also the review paper by T. Janssen, Acta Cryst. A42, 261 (1986).

[4] R. Penrose, Bull Inst Maths its Appl 10 n 7/8, 266 (1974); ibid. Math. Intelligencer 2, 32 (1979); see also M. Gardner, Sci. Amer. 236, 110 (1977)

[5] A. L. Mackay, Sov. Phys. Crystallogr. 26(5), 517 (1981); ibid. Physica 114A, 609 (1982), North-Holland Publishing Co.,

[6] N. G. de Bruijn, Kon. Nederl. Akad. Wetensch. Proceedings Ser. A 84 (Indag. Math. 43), 38 (1981).

[7] P. Kramer and R. Neri in: Acta Cryst. A 40, 580 (1984).

[8] D. Levine and P. J. Steinhardt, PRL 53, 2477 (1984).

[9] M. Duneau M and A. Katz, PRL 54, 2688 (1985); A. Katz and M. Duneau, J. de Physique 47, 181 (1986); P.A. Kalugin, A.Y. Kitayev and L. S. Levitov, J. Physique Lett. 46 L, 601(1985); V. Elser, Acta Cryst. A 42, 36 (1986).

[10] L. Pauling, Nature 317, 512 (1985); "Pauling's model not universally accepted", answers to L. Pauling, Nature 319, 102 (1986).

[11] L. Bendersky, PRL 55, 1461 (1985); B. Dubost et al., Nature 324, 48 (1986); A.P. Tsai et al., J. Mater. Sci. Lett. 6, 1403 (1987). See also A.P. Tsai et al., Phil. Mag. Lett. 61-1, 9 (1990).

\section{Further reading:}

[12] The Physics of Quasicrystals, editors P. J. Steinhardt and S. Ostlund, World Scientific, Singapore, (1987).

[13] Quasicrystals: a primer, C. Janot, Oxford University Press, Oxford (1992). 